(C) The Authors 2016. This is an Open Access article, distributed under the terms of the Creative

Commons Attribution licence (http://creativecommons.org/licenses/by/4.0/), which permits unrestricted

re-use, distribution, and reproduction in any medium, provided the original work is properly cited

\title{
Identification of (poly)phenol treatments that modulate the release of pro-inflammatory cytokines by human lymphocytes
}

\author{
Christopher T. Ford ${ }^{1} \dagger$, Siân Richardson ${ }^{1} \dagger$, Francis McArdle $^{1}$, Silvina B. Lotito ${ }^{2}$, Alan Crozier ${ }^{3}$, \\ Anne McArdle ${ }^{1}$ and Malcolm J. Jackson ${ }^{1}$. \\ ${ }^{1}$ Department of Musculoskeletal Biology, Institute of Ageing and Chronic Disease, University of Liverpool, Liverpool L7 8TX, UK \\ ${ }^{2}$ Unilever, Colworth Science Park, Sharnbrook, Bedford MK44 1LQ, UK \\ ${ }^{3}$ Department of Nutrition, University of California, Davis, CA 95616, USA
}

(Submitted 23 July 2015 - Final revision received 22 January 2016 - Accepted 12 February 2016 - First published online 17 March 2016)

\section{Abstract}

Diets rich in fruits and vegetables (FV), which contain (poly)phenols, protect against age-related inflammation and chronic diseases. T-lymphocytes contribute to systemic cytokine production and are modulated by FV intake. Little is known about the relative potency of different (poly)phenols in modulating cytokine release by lymphocytes. We compared thirty-one (poly)phenols and six (poly)phenol mixtures for effects on pro-inflammatory cytokine release by Jurkat T-lymphocytes. Test compounds were incubated with Jurkat cells for $48 \mathrm{~h}$ at 1 and $30 \mu \mathrm{m}$, with or without phorbol ester treatment at $24 \mathrm{~h}$ to induce cytokine release. Three test compounds that reduced cytokine release were further incubated with primary lymphocytes at 0.2 and $1 \mu \mathrm{m}$ for $24 \mathrm{~h}$, with lipopolysaccharide added at $5 \mathrm{~h}$. Cytokine release was measured, and generation of $\mathrm{H}_{2} \mathrm{O}_{2}$ by test compounds was determined to assess any potential correlations with cytokine release. A number of (poly) phenols significantly altered cytokine release from Jurkat cells $(P<0.05)$, but $\mathrm{H}_{2} \mathrm{O}_{2}$ generation did not correlate with cytokine release. Resveratrol, isorhamnetin, curcumin, vanillic acid and specific (poly)phenol mixtures reduced pro-inflammatory cytokine release from T-lymphocytes, and there was evidence for interaction between (poly)phenols to further modulate cytokine release. The release of interferon$\gamma$ induced protein 10 by primary lymphocytes was significantly reduced following treatment with $1 \mu$ m isorhamnetin $(P<0 \cdot 05)$. These results suggest that (poly)phenols derived from onions, turmeric, red grapes, green tea and açai berries may help reduce the release of proinflammatory mediators in people at risk of chronic inflammation.

Key words: Jurkat cells: Curcumin: Resveratrol: Isorhamnetin: TNF $\alpha$

(Poly)phenols, comprising flavonoids and related compounds, are produced by plants that are widely consumed in the human diet. A high dietary intake of fruits and vegetables, which are rich in (poly)phenols, has been linked through epidemiological studies with reduced risk for diseases that are associated with age-related chronic inflammation, including $\mathrm{CVD}^{(1-3)}$, type II diabetes ${ }^{(4,5)}$, cancers $^{(6-9)}$, Alzheimer's disease ${ }^{(10)}$ and Parkinson's disease ${ }^{(11)}$. Supplementation with (poly)phenolrich foods or purified (poly)phenols has been reported to reduce levels of pro-inflammatory cytokines in the human circulation, including TNF $\alpha$ and IL-6, cytokines that have been implicated in the pathogenesis of chronic inflammatory diseases $^{(12-14)}$. T-lymphocytes significantly contribute to the release of pro- and anti-inflammatory cytokines both into the circulation and within tissues. Dietary (poly)phenols may influence the behaviour of T-lymphocytes and other cells via redox signalling, that is the production or quenching of free radicals, or by interactions with specific proteins ${ }^{(15)}$. The production of $\mathrm{H}_{2} \mathrm{O}_{2}$ by (poly)phenols through autoxidation catalysed by transition metals is known to influence the behaviour of cells in culture ${ }^{(16,17)}$, but the effects of (poly)phenolinduced $\mathrm{H}_{2} \mathrm{O}_{2}$ generation on T-lymphocytes and the relevance of this in the modulation of cytokine release are not well understood. A majority of (poly)phenols have low bioavailability in vivo as parent compounds ${ }^{(18,19)}$; therefore, recent interest has been focused on metabolites of dietary (poly) phenols, including the methylated, glucuronidated, and sulphated conjugates that are produced by intestinal and liver cells, and the aromatic acids produced by the colonic bacterial flora ${ }^{(20-22)}$.

We aimed to compare thirty-one individual (poly)phenols for their effects on the release of inflammatory cytokines by Jurkat $\mathrm{CD}^{+}$T-lymphocytes. We examined some key dietary (poly)

Abbreviations: DMSO, dimethyl sulfoxide; LPS, lipopolysaccharide; PBMC, peripheral blood mononuclear cell; PHA, phytohaemagglutinin; PMA, phorbol 12-myristate 13-acetate; RPMI, Roswell Park Memorial Institute.

* Corresponding author: Professor M. J. Jackson, email mjj@liverpool.ac.uk

$\dagger$ Both authors contributed equally to this work 
phenols and a number of metabolites, including specifically a substantial number of low molecular weight colonic catabolites. Following this initial screen, three anti-inflammatory compounds were selected for further investigation of their effects on cytokine release by primary peripheral blood mononuclear cell (PBMC)-derived human lymphocytes. We further aimed to assess the generation of $\mathrm{H}_{2} \mathrm{O}_{2}$ in cell culture media by these (poly)phenols and to assess whether $\mathrm{H}_{2} \mathrm{O}_{2}$ generation significantly influences cytokine release or growth of Jurkat $\mathrm{CD}^{+}{ }^{+} \mathrm{T}$-lymphocytes in vitro. The question of the most appropriate doses of (poly)phenols to be used in in vitro studies is controversial within the field. Following dietary intake, (poly)phenols and the individual metabolites/catabolites can be detected in the circulation with $C_{\max }$ values generally at nм or low $\mu \mathrm{m}$ concentrations, whereas following supplementation with purified compounds or enriched food extracts values of up to $20-50 \mu \mathrm{m}$ have been reported. For an individual with a typical dietary intake of multiple fruits and vegetables, numerous (poly)phenol metabolites/catabolites coexist in the circulation at any one time. Whether mixtures of different (poly) phenols, each present at low doses, interact in their effects on cytokine release by lymphocytes has not been clear. We therefore also investigated the effects of six different mixtures of (poly)phenols on the release of pro-inflammatory cytokines by Jurkat T-lymphocytes, in order to determine whether multiple (poly)phenols at lower doses have additive or potentially synergistic effects compared with those of single (poly)phenols.

\section{Methods}

\section{Chemicals and materials}

Reagents were purchased from Sigma-Aldrich unless stated otherwise. Sigma-Aldrich also supplied resveratrol, quercetin, isorhamnetin, 3-O-methylquercetin, curcumin, (-)-epigallocatechin3-O-gallate, pelargonidin-3-O-glucoside, cyanidin-3-Oglucoside, chlorogenic acid (5-O-caffeoylquinic acid), punicalagin, phloroglucinol (1,3,5-trihydroxybenzene), pyrogallol (1,2,3trihydroxybenzene), catechol (1,2-dihydroxybenzene), protocatechuic acid (3,4-dihydroxybenzoic acid), 4-hydroxybenzoic acid, homoprotocatechuic acid (3',4'-dihydroxyphenylacetic acid), vanillic acid (3-methoxy-4-hydroxybenzoic acid), homovanillic acid (3'-methoxy-4'-hydroxyphenylacetic acid), 4'-hydroxyphenylacetic acid, 4'-hydroxymandelic acid, 5-(3'hydroxyphenyl) propionic acid, 3-(4'-hydroxyphenyl) lactic acid, caffeic acid, dihydrocaffeic acid (3-(3',4'-dihydroxyphenyl) propionic acid), ferulic acid, isoferulic acid, dihydroferulic acid (3-(3'-methoxy-4'-hydroxyphenyl) propionic acid), hippuric acid and tyrosol. 4'-Hydroxyhippuric acid was obtained from Bachem Ltd. Feruloylglycine and isoferuloylglycine were generous gifts from Professor Takao Yokota (Teikyo University). Fig. 1 shows the structures of the compounds tested and highlights the metabolic relationships between the compounds.

\section{Cell culture and treatments}

Jurkat E6.1 human $\mathrm{CD}^{+}$T-lymphocytes obtained from the Health Protection Authority, UK, were cultured routinely at $37^{\circ} \mathrm{C}$ in a humidified $5 \% \mathrm{CO}_{2}$ incubator in Roswell Park Memorial Institute (RPMI)-1640 medium containing phenol red supplemented with $10 \%$ fetal bovine serum (FBS) and 2 mM-Lglutamine. Jurkat cells were seeded at $2 \times 10^{6}$ cells $/ \mathrm{ml}$ in a fortyeight-well plate $(500 \mu \mathrm{l}$ culture volume) and treated with each test compound at 1 or $30 \mu \mathrm{mol} / 1$ by dilution from $5 \mathrm{~mm}$ stocks in dimethyl sulfoxide (DMSO). The same volume of DMSO only was added to vehicle control wells. After $24 \mathrm{~h}$ of incubation with test compounds, $25 \mathrm{ng} / \mathrm{ml}$ phorbol 12-myristate 13-acetate (PMA) by 1:1000 dilution from a $25 \mu \mathrm{g} / \mathrm{ml}$ DMSO stock solution and $5 \mu \mathrm{g} / \mathrm{ml}$ phytohaemagglutinin (PHA) by 1:1000 dilution from a $5 \mathrm{mg} / \mathrm{ml}$ stock solution in water were added to some wells, and vehicle controls were treated with DMSO at 1:1000 dilution. Typically, the equivalent volume of DMSO added to each well was $0.5 \mu \mathrm{l}$. Co-treatment with PMA (a protein kinase C activator) and PHA (a T-cell receptor cross-linking agent) has been widely used to stimulate cytokine production by T-lymphocytes $^{(23)}$. Following PMA/PHA treatment, plates were incubated for $24 \mathrm{~h}$ at $37^{\circ} \mathrm{C}$ and $5 \% \mathrm{CO}_{2}$. Test compounds were not removed during PMA/PHA treatment, and thus the total incubation period with each (poly)phenol was $48 \mathrm{~h}$. Each treatment was performed in quadruplicate alongside matched vehicle controls. Mixed (poly)phenol treatments were conducted using the same protocol, except that each of the four compounds in each mixture was added at either 0.25 or $7.5 \mu \mathrm{m}$, thus achieving either a 1 or $30 \mu \mathrm{m}$ total concentration of (poly)phenols.

\section{Measurement of viable cell number}

Following incubations with test compounds and stimulation agents, cell numbers were quantified by transferring $100 \mu \mathrm{l}$ cell suspension from each well to a flat-bottomed, clear, ninetysix-well plate. A sample of $20 \mathrm{ml}$ of 3-(4,5-dimethylthiazol-2-yl)5-(3-carboxymethoxyphenyl)-2-(4-sulfophenyl)-2H-tetrazolium, inner salt and phenazine methosulfate reagent (Promega) was added to each well and the plate was incubated for $90 \mathrm{~min}$ at $37^{\circ} \mathrm{C}$ and $5 \% \mathrm{CO}_{2}$. Following incubation, absorbance was read at $490 \mathrm{~nm}$ on a BMG Labtech Spectrostar Nano plate reader. Viable cell numbers were estimated from a standard curve determined by haemocytometer counting with $0.02 \%$ $(\mathrm{w} / \mathrm{v})$ trypan blue stain.

\section{Primary lymphocyte isolation, cell culture and treatment}

Primary human lymphocytes were obtained from a healthy 45-year-old female donor after obtaining written consent, by withdrawal of $50 \mathrm{ml}$ blood from the antecubital vein using a butterfly needle and syringe. The procedure was approved by the National Research Ethics service, UK (reference 11/NW/ 0313), as well as by the University of Liverpool and Royal Liverpool and Broadgreen University Hospitals NHS Trust ethics committees. Whole blood was separated over Ficoll-Paque Premium density gradient medium (GE Healthcare Life Sciences) by centrifugation at $370 \boldsymbol{g}$ for $30 \mathrm{~min}$ without rotor braking. PBMC were isolated from the buffy layer, centrifuged again at $370 \boldsymbol{g}$ for $10 \mathrm{~min}$ without rotor braking, then resuspended in FBS containing 10\% DMSO and cryogenically stored. The PBMC were later thawed, washed once in medium 
(Poly)phenols modulate T-lymphocyte cytokine release

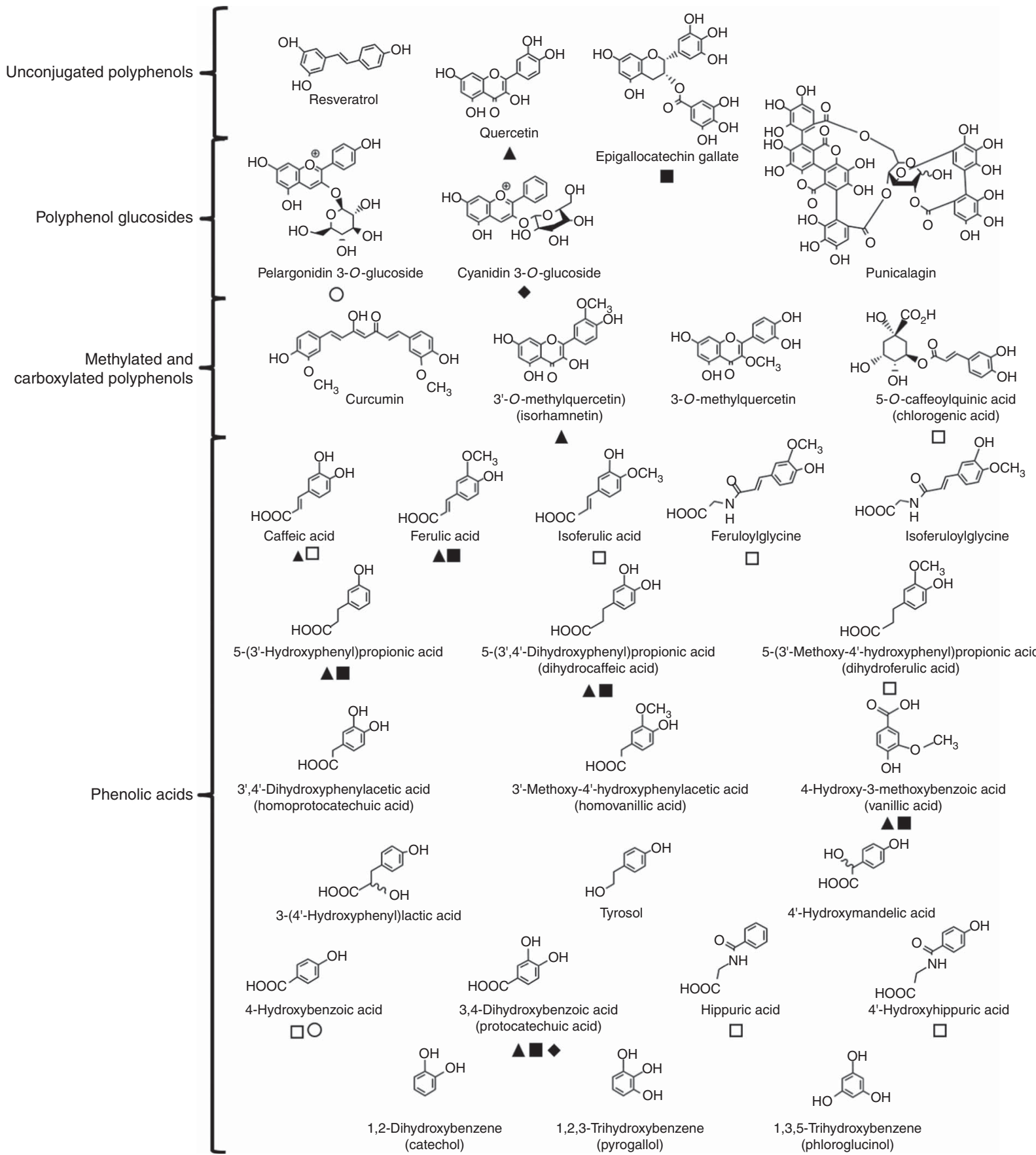

Fig. 1. Structures of (poly)phenols used in cell culture experiments. Confirmed metabolic relationships are shown: $\mathbf{\Delta}$, metabolite of quercetin ${ }^{(23,24)}$; $(-)$-epigallocatechin-3-O-gallate ${ }^{(25,26)} ;$, metabolite of cyanidin-3-O-glucoside ${ }^{(27)} ; O$, metabolite of pelargonidin-3-O-glucoside ${ }^{(28)}$; and $\square$, metabolite of chlorogenic acid $^{(29)}$. The phenolic catabolite structures are approximately ordered from those produced in the small intestine (top) to those derived in the proximal gastrointestinal tract via colonic catabolism (bottom).

(RPMI-1640 supplemented with $10 \%$ FBS and $2 \mathrm{mmol} / \mathrm{l}$ of L-glutamine) to remove DMSO, resuspended in the same medium and then cultured at $37^{\circ} \mathrm{C}$ and $5 \% \mathrm{CO}_{2}$ in a T25 tissue culture flask laid horizontally. After $5 \mathrm{~d}$ of incubation to allow monocytes to adhere to the culture flask, the lymphocyteenriched suspension cells were seeded to a forty-eight-well plate at $2 \times 10^{6}$ cells $/ \mathrm{ml}$ in $150 \mu \mathrm{l}$ total volume. After $24 \mathrm{~h}$ of incubation at $37^{\circ} \mathrm{C} / 5 \% \mathrm{CO}_{2}$, the lymphocytes were treated with test compounds (resveratrol, isorhamnetin and curcumin) diluted from $5 \mathrm{mmol} / \mathrm{l}$ DMSO stocks to a final concentration of either 0.2 or $1 \mu \mathrm{mol} / 1$, or the same volume of DMSO only as the vehicle control. Lymphocytes were incubated for $5 \mathrm{~h}$ at $37^{\circ} \mathrm{C} / 5 \% \mathrm{CO}_{2}$; next, $20 \mathrm{ng} / \mathrm{ml}$ lipopolysaccharide (LPS) was added without removing media and the cells were incubated for a further $19 \mathrm{~h}$. Following the total $24 \mathrm{~h}$ of incubation, cell numbers and viability were determined by trypan blue staining 
$(0.02 \% \mathrm{w} / \mathrm{v}$ final concentration trypan blue; TC-20 automated cell counter, Bio-Rad) and media were harvested for quantification of cytokines.

\section{Quantification of cytokines in lymphocyte cell culture media}

Cells were pelleted by centrifugation at $400 \boldsymbol{g}$ for $5 \mathrm{~min}$. The culture media supernatants were immediately frozen at $-80^{\circ} \mathrm{C}$ and remained frozen until they were thawed for analysis using a BioPlex 200 multi-protein analysis platform (Bio-Rad) and BioPlex bead sets targeting the human cytokine analytes detailed in Table 1 . The analysis was performed according to the manufacturer's instructions. All reagents were purchased from Bio-Rad.

Cytokine concentrations were calculated by comparing raw fluorescence emission values with a standard curve diluted from a solution of mixed recombinant cytokine proteins (Bio-Rad). Cytokine concentrations were then normalised for the number of viable cells in the culture at the end of treatment, as quantified by trypan blue exclusion assay. Each time a Jurkat cell treatment experiment was performed, four test compounds were assayed together with a matched vehicle control (either DMSO alone or DMSO with PMA/PHA stimulation at $24 \mathrm{~h}$, as appropriate). Data were normalised to yield percentage changes from vehicle controls by dividing values measured from (poly)phenol-treated cells by the value measured from the matched vehicle control and multiplying by 100 . To ascertain whether mixtures of (poly)phenols may synergistically modulate Jurkat T-lymphocyte cytokine release, six mixtures were analysed, each comprised of four (poly)phenols. The results obtained following treatment of Jurkat T-lymphocytes with the (poly)phenol mixtures were compared with the averaged effects of those individual compounds. The total concentration of mixed (poly)phenols and their metabolites was constant at 1 or $30 \mu \mathrm{mol} / 1$ to enable closer comparison with the individual compound treatments.

\section{Luminescent plate reader analysis of $\mathrm{H}_{2} \mathrm{O}_{2}$ generation}

Using an opaque, black, ninety-six-well plate, 5 mm-DMSO stock solutions of thirty-two different (poly)phenols (the same thirty-one compounds tested for anti-inflammatory effects in the Jurkat cell model, plus homovanillic acid) were diluted to a final concentration of $30 \mu \mathrm{m}$ in $200 \mu \mathrm{l}$ of working solution (1:1 solution of RPMI-1640 and Amplex Red (10-acetyl-3,7dihydroxyphenoxazine)-HRP reagent; Invitrogen). A DMSOonly control was used as blank. Fluorescence was measured at 5-min intervals for $24 \mathrm{~h}$ with a Fluostar Omega plate reader (BMG Labtech) using an excitation wavelength of $570 \mathrm{~nm}$ and an emission wavelength of $585 \mathrm{~nm}$. Kinetic reactions were quantified from the gradient of the linear phase of each reaction and converted into the rates of $\mathrm{H}_{2} \mathrm{O}_{2}$ generation $(\mathrm{nmol} / \mathrm{min}$ ) by comparison with $\mathrm{H}_{2} \mathrm{O}_{2}$ standards.

\section{Statistical analysis}

Statistical analyses were performed using SPSS version 20 (IBM) using raw data for cell number and $\mathrm{H}_{2} \mathrm{O}_{2}$ generation and data normalised to cell number for cytokine measurements. Jurkat
$\mathrm{CD}^{+}$T-lymphocytes and primary PBMC-derived lymphocytes treated with (poly)phenols were compared with matched vehicle controls by one-way ANOVA with Dunnett's post hoc test. The predicted effects of mixtures of test compounds on cytokine release by Jurkat $\mathrm{CD}^{+}{ }^{+} \mathrm{T}$-lymphocytes were compared with the observed effects by one-way ANOVA with Tukey's post hoc test. All the statistical tests were performed at $\alpha=0.05$.

\section{Results}

Phorbol 12-myristate 13-acetate/phytohaemagg/utinin 24-h treatment stimulates the release of pro-inflammatory cytokines by Jurkat CD4 ${ }^{+}$T-lymphocytes

Jurkat CD4 + T-lymphocytes were treated with the protein kinase $\mathrm{C}$ activator PMA and the plant-derived lymphocyte mitogen PHA for $24 \mathrm{~h}$ to induce an inflammatory response and cytokine release. The PMA/PHA-stimulated cells showed a significant increase in the release of several pro-inflammatory cytokines compared with vehicle control treatments (Table 1). We chose to focus on IL-2, IL- 8 and TNF $\alpha$ for further experiments because of the relative magnitude of their induction by PMA/ PHA treatment and the well-defined roles of each of these proteins as physiologically important pro-inflammatory cytokines.

\section{Polyphenol catabolites modulate growth and cytokine release by Jurkat $C D 4^{+} T$-lymphocytes}

After treatment with colonic catabolites of polyphenols (phenolic acids) for $48 \mathrm{~h}$, we measured the density of viable Jurkat $\mathrm{CD}^{+}$T-lymphocytes in culture and the concentrations of the pro-inflammatory cytokines IL-2, IL- 8 and TNF $\alpha$ in the cell culture media. Comparisons between (poly)phenol treatments and vehicle controls revealed multiple effects on growth and cytokine release (Fig. 2). The low molecular weight $(110-139 \mathrm{~g} / \mathrm{mol})$ catabolites catechol, phloroglucinol and 4-hydroxybenzoic acid caused significant declines in cell number $(P<0 \cdot 05$; one-way ANOVA with Dunnett's post hoc test $)$ and significantly induced release of IL-2, IL- 8 and TNF $\alpha(P<0 \cdot 05$; oneway ANOVA with Dunnett's post hoc test), whereas tyrosol significantly decreased IL-2 and IL-8 release from non-stimulated cells at $1 \mu \mathrm{mol} / 1(P<0.05$; one-way ANOVA with Dunnett's post hoc test). Mid-molecular weight catabolites $(168-195 \mathrm{~g} / \mathrm{mol})$ reduced pro-inflammatory cytokine release, with significant reduction in IL-2 release from non-stimulated cells by $1 \mu \mathrm{mol} / 1$ 4'-hydroxymandelic acid and vanillic acid $(P<0.05$; one-way ANOVA with Dunnett's post hoc test). Higher molecular weight test compounds (196-252 g/mol) showed mixed effects: feruloylglycine at $1 \mu \mathrm{mol} / 1$ significantly increased the release of IL- 2 and $\mathrm{TNF} \alpha$, and isoferuloylglycine at $30 \mu \mathrm{mol} / 1$ significantly reduced IL-8 release from PMA/PHA-stimulated Jurkat $\mathrm{CD}^{+}$T-lymphocytes $(P<0 \cdot 05$; one-way ANOVA with Dunnett's post hoc test).

\section{Polyphenols modulate growth and pro-inflammatory cytokine release by Jurkat CD $4^{+}$T-lymphocytes}

Treatment with different polyphenols had varied effects on growth and pro-inflammatory cytokine release by Jurkat $\mathrm{CD}^{+}$ 
Table 1. Cytokines induced by phorbol 12-myristate 13-acetate/phytohaemagglutinin (PMA/PHA) treatment of Jurkat CD4 ${ }^{+}$-lymphocytes (Mean values with their standard errors)

\begin{tabular}{|c|c|c|c|c|c|c|c|}
\hline \multirow[b]{2}{*}{ Cytokines } & \multicolumn{3}{|c|}{ Untreated control (pg/ml) } & \multicolumn{2}{|c|}{ DMSO vehicle control (pg/ml) } & \multicolumn{2}{|c|}{$24 \mathrm{~h}$ PMA/PHA stimulated (pg/ml) } \\
\hline & Mean & & SEM & Mean & SEM & Mean & SEM \\
\hline Eotaxin-1 & & ND & & 2.85 & 1.47 & 0.48 & 0.56 \\
\hline GM-CSF & & ND & & & & $5 \cdot 81^{*}$ & 0.39 \\
\hline IFN- $\gamma$ & & ND & & & & & \\
\hline $\mathrm{IL}-1 \beta$ & & ND & & & & & \\
\hline $\mathrm{IL}-2$ & 13.45 & & 1.39 & 14.40 & 1.41 & $6969.69^{*}$ & 303.95 \\
\hline IL-4 & 21.55 & & 0.35 & & & 1.86 & 5.67 \\
\hline IL-6 & & ND & & & & & \\
\hline IL-7 & 0.3 & & 0.06 & 0.80 & 0.66 & 3.52 & 0.66 \\
\hline IL-8 & $35 \cdot 25$ & & 2.88 & $34 \cdot 22$ & 2.32 & $1612 \cdot 68^{\star}$ & $92 \cdot 71$ \\
\hline IL-12p70 & & ND & & & & & \\
\hline IL-13 & & ND & & & & $7 \cdot 68^{*}$ & 1.52 \\
\hline MCP-1 & & ND & & & & & \\
\hline TNFa & & ND & & & & $259.55^{\star}$ & $12 \cdot 36$ \\
\hline
\end{tabular}

GM-CSF, granulocyte macrophage-colony stimulating factor; IFN, interferon; MCP, monocyte chemoattractant protein.

* $P<0.05$ compared with DMSO vehicle control.

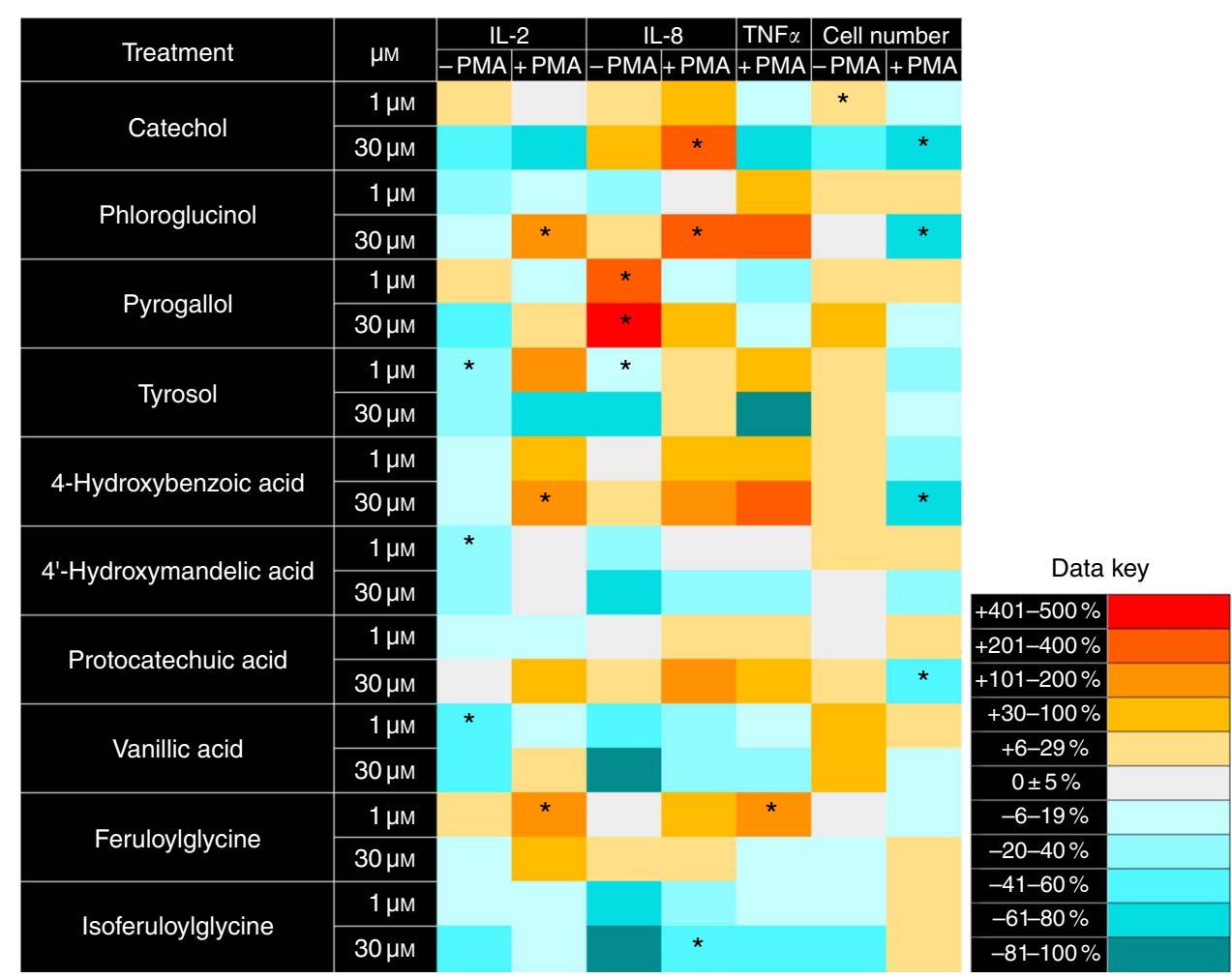

Fig. 2. Heat map showing the effects of phenolic acids on cytokine release and growth by Jurkat CD4 ${ }^{+}$T-lymphocytes. Compounds are ordered by molecular weight from the lowest weight (top) to the highest weight (bottom). Data are presented as percentage differences from matched vehicle controls following $48 \mathrm{~h}$ of treatment. Treatment and control experiments were performed with or without $25 \mathrm{ng} / \mathrm{ml}$ phorbol 12-myristate 13-acetate (PMA) and $5 \mu \mathrm{gg} / \mathrm{ml}$ phytohaemagglutinin (PHA) stimulation at $24 \mathrm{~h}$. TNF $a$ could not be measured in the absence of PMA/PHA stimulation. * Mean value was significantly different compared with vehicle controls $(P<0.05$; one-way ANOVA with Dunnett's post hoc test). No significant effects were observed following treatment with caffeic acid, ferulic acid, isoferulic acid, 5-(3'-hydroxyphenyl) propionic acid, 5-(3',4'-dihydroxyphenyl) propionic acid, 5-(3'-methoxy-4'-hydroxyphenyl) propionic acid, homoprotocatechuic acid, 3-(4' hydroxyphenyl) lactic acid, hippuric acid or 4'-hydroxyhippuric acid. Examples of the data from which these heat maps are derived are provided in the online Supplementary data to allow an assessment of the variability observed in these studies.

T-lymphocytes (Fig. 3). Three polyphenols - resveratrol, isorhamnetin and curcumin - significantly reduced pro-inflammatory cytokine release at both 1 and $30 \mu \mathrm{mol} / \mathrm{l}$ and in both unstimulated and PMA/PHA-stimulated cells $(P<0.05$; one-way ANOVA with Dunnett's post hoc test). Curcumin treatment at $30 \mu \mathrm{mol} / \mathrm{l}$ led to the greatest reductions in pro-inflammatory cytokine release, with IL-2 release decreased by $96 \%$ and $\mathrm{TNF} \alpha$ release ablated to undetectable 


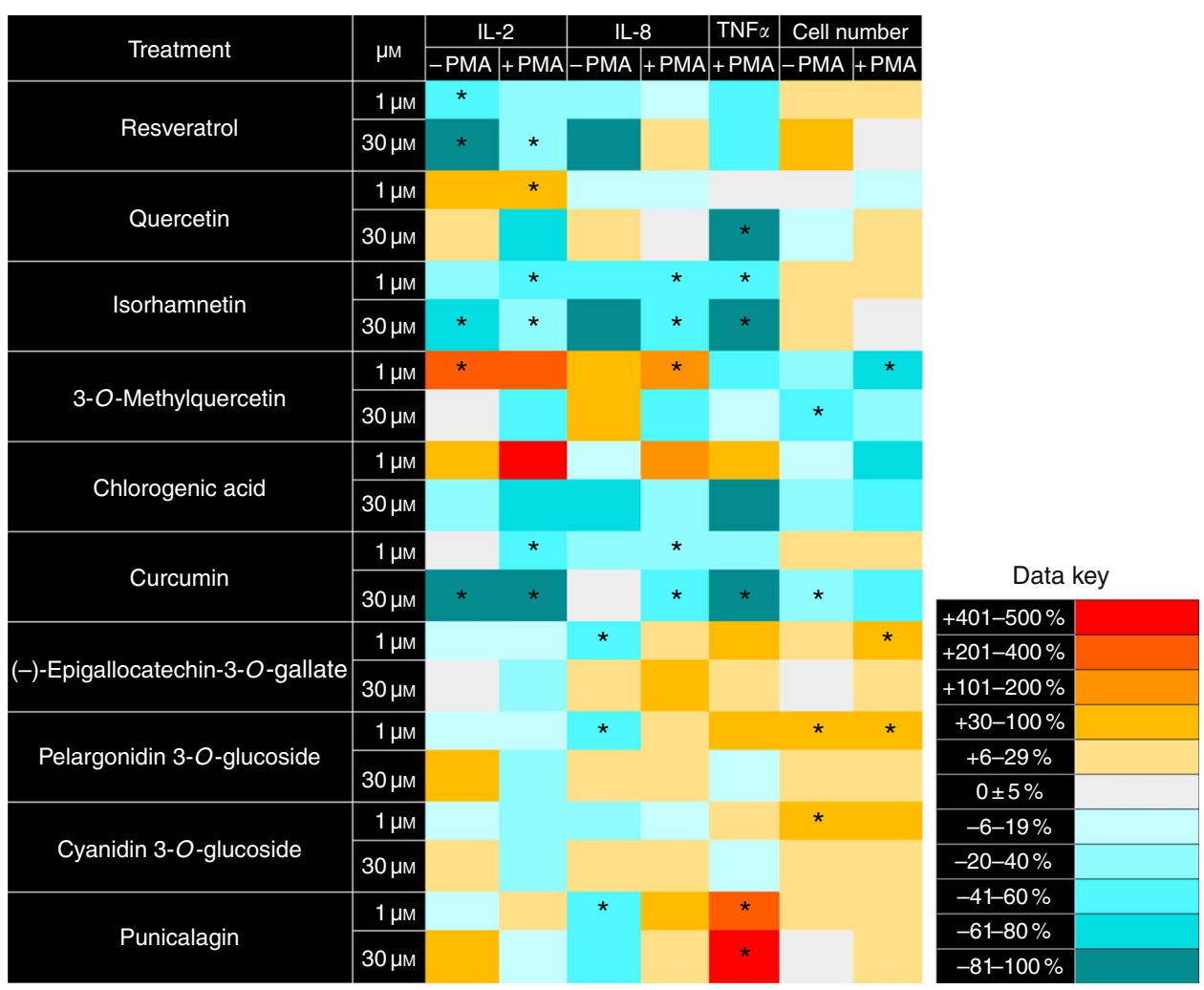

Fig. 3. Heat map showing the effects of polyphenols on cytokine release and growth by Jurkat CD4 ${ }^{+}$T-lymphocytes. Compounds are ordered by molecular weight from the lowest weight (top) to the highest weight (bottom). Data are presented as percentage differences from matched vehicle controls following $48 \mathrm{~h}$ of treatment. Treatment and control experiments were performed with or without $25 \mathrm{ng} / \mathrm{ml}$ phorbol 12-myristate 13-acetate (PMA) and $5 \mu \mathrm{g} / \mathrm{ml}$ phytohaemagglutinin (PHA) stimulation at $24 \mathrm{~h}$. TNF $a$ could not be measured in the absence of PMA/PHA stimulation. * Mean value was significantly different compared with vehicle controls $(P<0.05$; one-way ANOVA with Dunnett's post hoc test). Examples of the data from which these heat maps are derived are provided in the online Supplementary data to allow an assessment of the variability observed in these studies.

concentrations in PMA/PHA-stimulated cells $(P<0.05$; one-way ANOVA with Dunnett's post hoc test). The flavan3-ol (-)-epigallocatechin-3-O-gallate, and the anthocyanins pelargonidin-3-O-glucoside and cyanidin 3-O-glucoside, significantly promoted the growth of Jurkat $\mathrm{CD}^{+}$ T-lymphocytes at $1 \mu \mathrm{mol} / \mathrm{l}(P<0.05$; one-way ANOVA with Dunnett's post hoc test). Chlorogenic acid and 3-Omethylquercetin showed some anti-proliferative effects on Jurkat $\mathrm{CD}^{+}$T-lymphocytes under all treatment conditions, although statistical significance was only achieved for the effects of 3-O-methylquercetin $(P<0 \cdot 05$; one-way ANOVA with Dunnett's post hoc test).

\section{Mixtures of (poly)phenols interact to modulate cytokine release}

To assess potential interactions between test compounds in modulating cytokine release, we prepared several mixtures of (poly)phenols: mixture 1 representing low-molecular weight colonic catabolites such as catechol, phloroglucinol, 4-hydroxybenzoic acid and protocatechuic acid; mixture 2 representing mid-molecular weight colonic catabolites such as 4'-hydroxymandelic acid, 4'-hydroxyphenylacetic acid, 5-(3'-hydroxyphenyl) propionic acid and 3-(4'-hydroxyphenyl) lactic acid; mixture 3 representing a mixture of dietary polyphenols such as (-)-epigallocatechin-3-O-gallate, pelargonidin-3-O-glucoside, cyanidin-3-O-glucoside and punicalagin; mixture 4 representing polyphenols and high molecular weight hydroxycinnamate metabolites, such as dihydroferulic acid, feruloylglycine, quercetin and 3-O-methylquercetin; mixture 5 representing a mixture of hydroxycinnamate derivatives derived from chlorogenic acid after the consumption of coffee, such as caffeic acid, ferulic acid, isoferulic acid and isoferuloylglycine; and mixture 6 representing compounds derived from apple cider, such as hippuric acid, tyrosol, 4'-hydroxyhippuric acid and chlorogenic acid. We measured cytokine release from Jurkat $\mathrm{CD}^{+}$T-lymphocytes following $48 \mathrm{~h}$ of incubation with each mixture at a total concentration of 1 or $30 \mu \mathrm{mol} / \mathrm{l}$, with or without stimulation with PMA/PHA at $24 \mathrm{~h}$. For comparison, the results previously obtained for cytokine release following treatment with each individual compound were averaged to generate a prediction of the effects expected if there were no interactions between compounds (i.e. a null hypothesis that there were no synergistic effects). The results illustrated in Fig. 4 indicate that five of the six test mixtures significantly reduced pro-inflammatory cytokine release in comparison with results anticipated from simple addition of the effects observed for individual compounds $(P<0 \cdot 05$; one-way ANOVA with Dunnett's post hoc test). 

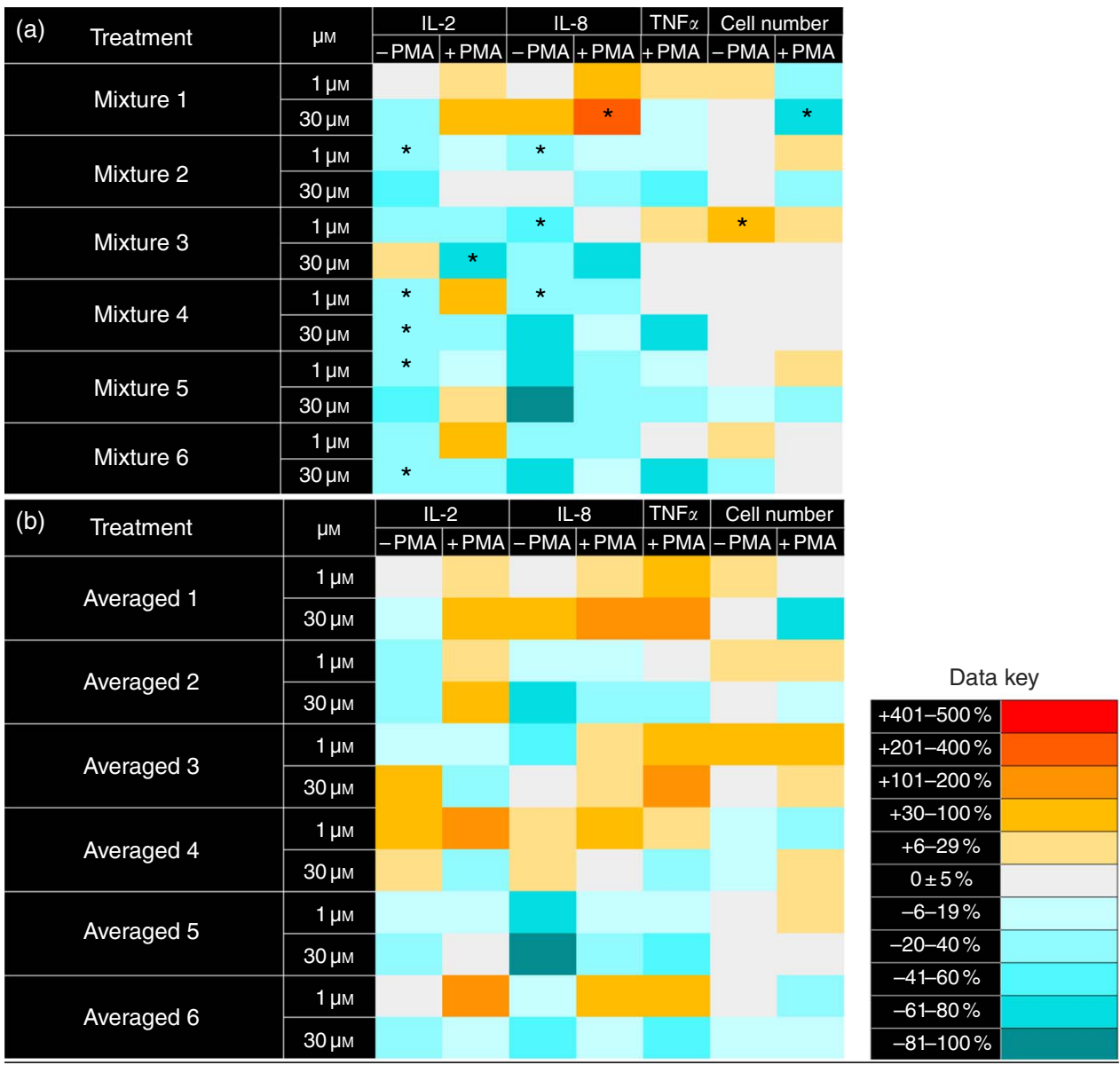

Fig. 4. Heat maps showing cytokine release by Jurkat CD4+ T-lymphocytes following treatment with mixtures of four (poly)phenols at 1 or $30 \mu \mathrm{mol} / /$ compared with vehicle controls (a), and mathematical averages of the effects on cytokine release following treatment with the individual compounds (b). Cells were incubated with (poly)phenol mixtures for $48 \mathrm{~h}$, with or without the addition of $25 \mathrm{ng} / \mathrm{ml}$ phorbol myristoyl acetate and $5 \mu \mathrm{g} / \mathrm{ml}$ phytohaemagglutinin at the 24-h time point. The mixtures comprised the following: (1) catechol, phloroglucinol, 4-hydroxybenzoic acid and protocatechuic acid; (2) 4'-hydroxymandelic acid, 4-hydroxyphenylacetic acid, 5-(3'-hydroxyphenyl) propionic acid and 3-(4'-hydroxyphenyl) lactic acid; (3) (-)-epigallocatechin-3-O-gallate, pelargonidin-3-O-glucoside, cyanidin-3-O-glucoside and punicalagin; (4) dihydroferulic acid, feruloylglycine, quercetin and 3-O-methylquercetin; (5) caffeic acid, ferulic acid, isoferulic acid and isoferuloylglycine; and (6) hippuric acid, tyrosol, 4'-hydroxyhippuric acid and chlorogenic acid. * Mean value was significantly different between the 'expected' response predicted by mathematically averaging the effects of treatment with individual compounds and the 'observed' response measured following treatment with the mixtures $(P<0.05$; one-way ANOVA with Tukey's post hoc test).

Anti-inflammatory polyphenols modulate cytokine release by peripheral blood mononuclear cell-derived human lymphocytes

We investigated the polyphenols resveratrol, isorhamnetin and curcumin, which had been identified to be the most effective in reducing cytokine release from among the panel of (poly)phenols that we screened in Jurkat $\mathrm{CD}^{+}{ }^{+} \mathrm{T}$-lymphocytes, to determine whether their inhibitory effects on pro-inflammatory cytokine release were sustained in PBMC-derived human lymphocytes (Fig. 5). The results suggest trends (i.e. $P<0 \cdot 09$ ) towards decreased IL-6, interferon- $\gamma$ induced protein 10 (IP-10) and TNF $\alpha$ release following treatment with resveratrol, isorhamnetin and curcumin at 0.2 or $1 \mu \mathrm{m}$. The reduction in IP-10 release after treatment with $1 \mu \mathrm{m}$ isorhamnetin was statistically significant $(P<0.05$, one-way ANOVA with Dunnett's post hoc test).

\section{(Poly)phenols produce $\mathrm{H}_{2} \mathrm{O}_{2}$ in cell culture media}

Some (poly)phenols have been reported to generate $\mathrm{H}_{2} \mathrm{O}_{2}$ in cell culture media ${ }^{(16,17)}$. To quantify $\mathrm{H}_{2} \mathrm{O}_{2}$ production, we conducted kinetic spectrophotometry assays using Amplex red reagent, which is converted to fluorescent resorufin following oxidation by $\mathrm{H}_{2} \mathrm{O}_{2}$. Production of $\mathrm{H}_{2} \mathrm{O}_{2}$ was detected for sixteen of the thirty-two test compounds (Fig. $6(\mathrm{a})) \cdot \mathrm{H}_{2} \mathrm{O}_{2}$ production was detected from the hydroxybenzene derivatives catechol and pyrogallol, the phenylacetic acid homoprotocatechuic acid, the hydroxycinnamates caffeic acid and dihydrocaffeic acid, and the ellagitannin punicalagin, whereas other test compounds produced no detectable levels of $\mathrm{H}_{2} \mathrm{O}_{2}$. Comparisons were also made to assess the effects of phenol red in the culture media, which showed that rates of $\mathrm{H}_{2} \mathrm{O}_{2}$ production were 24 (SEM 2) \% lower in RPMI-1640 medium containing phenol red than in RPMI-1640 medium without phenol red (data not shown in detail). 


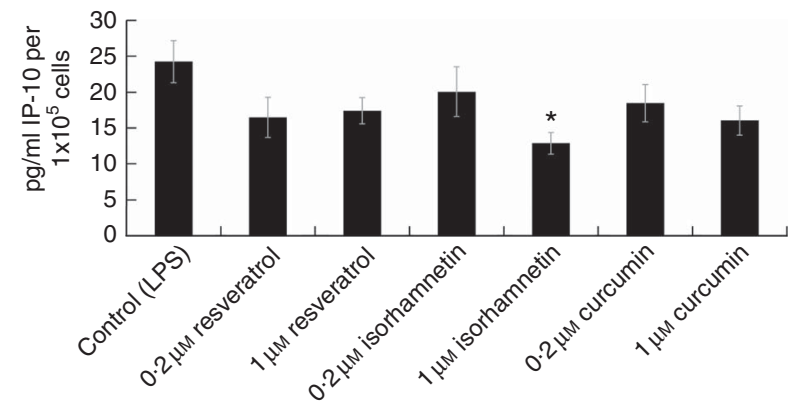

Fig. 5. Cytokine release by lymphocytes from a healthy donor following treatment for $24 \mathrm{~h}$ with the polyphenols resveratrol, isorhamnetin and curcumin with the addition of $20 \mathrm{ng} / \mathrm{ml}$ lipopolysaccharide (LPS) at the 5-h time point. Isorhamnetin at $1 \mu \mathrm{mol} / \mathrm{l}$ significantly reduced interferon- $\gamma$ induced protein 10 (IP-10) release compared with the matched DMSO vehicle control $(P<0.05$; one-way ANOVA with Dunnett's post hoc test). *Groups that significantly differed from the control group (one-way ANOVA with Dunnett's post hoc test; $P<0.05)$. There were no significant differences between groups for other cytokines. Data for the other cytokines are available in the Online Supplementary Fig. S2. LVC, LPS and vehicle control; 0.2 and 1 denote concentrations in $\mu \mathrm{mol} / \mathrm{l}$.

\section{Production of $\mathrm{H}_{2} \mathrm{O}_{2}$ by (poly)phenols does not strongly correlate with cytokine release by $C D 4^{+} T$-lymphocytes}

Correlation assays were performed between the $\mathrm{H}_{2} \mathrm{O}_{2}$ production by test compounds and their effects on Jurkat $\mathrm{CD} 4^{+}$ T-lymphocyte growth and pro-inflammatory cytokine release (Fig. 6(b)-(e)). These data indicate a potential correlation between $\mathrm{H}_{2} \mathrm{O}_{2}$ production and IL-8 release in non-PMA/ PHA-stimulated cells $\left(R^{2} 0 \cdot 3048\right)$.

\section{Discussion}

In these studies, we evaluated a panel of thirty-one (poly) phenols for potential anti-inflammatory activity using a human T-lymphocyte model of cytokine production. The compounds tested were grouped by their molecular weight (Fig. $2 \& 3$ ), as the lower molecular weight compounds tended to be the colonic catabolites (phenolic acids). We observed substantial inhibition of cytokine release primarily by the parent polyphenols, several of which modified cytokine release and proliferation by T-lymphocytes under both baseline and activated (PMA/PHA stimulated) conditions. We also investigated mixtures of (poly)phenols, and our data show that five of the six mixtures of test compounds had greater anti-inflammatory effects than that predicted under our null hypothesis based on the effects of the individual compounds. We further explored the effects of three of the dietary polyphenols showing anti-inflammatory effects in Jurkat cells in healthy human lymphocytes and found that isorhamnetin reduced pro-inflammatory cytokine release from LPS-stimulated lymphocytes at $1 \mu \mathrm{m}$. (Poly)phenols have been proposed to reduce the risk of developing chronic diseases during ageing by modulating inflammatory responses and the production of pro-inflammatory cytokines in multiple tissues ${ }^{(25)}$.

A number of previous studies have examined the potential effects of various polyphenols on cytokine release ${ }^{(28-31)}$, and a number of dietary components including curcumin, resveratrol, genistein and epigallocatechin have been shown to modulate the release of pro-inflammatory cytokines from cells in culture $^{(30)}$. Various mechanisms of action have been proposed including inhibition of $\mathrm{NF}-\kappa \mathrm{B}$, inhibition of prostanoids, inhibition of AMP-activated protein kinase (AMPK) and mitogenactivated protein kinase (MAPK) pathways and antioxidant effects $^{(31)}$. There has been a great deal of interest in the bioavailability of dietary polyphenols, as the non-metabolised compounds are only found in the circulation at nм to low $\mu \mathrm{M}$ concentrations. Compounds such as flavonoids are absorbed in the small intestine and appear in the circulation as glucuronoids, sulphates and methylated metabolites, but these are rapidly removed from the bloodstream ${ }^{(32)}$; however, substantial amounts of the unconjugated compounds pass into the colon where they are converted to lower molecular weight catabolites such as phenolic acids by colonic microflora ${ }^{(33)}$. Relatively little is known about the potential anti-inflammatory effects of these lower molecular weight phenolic acids following absorption, and this was a major aim of the present study.

Polyphenols were routinely examined at final concentrations of either 1 or $30 \mu \mathrm{m}$, reflecting concentrations that may potentially be achieved in the circulation through ingestion of foodstuffs or supplements, respectively. Our data showed that several (poly)phenol treatments modulated the release of pro-inflammatory cytokines (IL-2, IL-8 and TNF $\alpha$ ) from Jurkat human $\mathrm{CD}^{+}$T-lymphocytes. The Jurkat cell line was originally derived from an adolescent male with acute lymphoblastic leukaemia and they are widely used as a model for proinflammatory cytokine release ${ }^{(34)}$. Jurkat $\mathrm{CD}^{+}$T-lymphocytes were incubated with test compounds at 1 and $30 \mu \mathrm{m}$ either nonstimulated or stimulated with PMA/PHA. We were interested to investigate both lower and higher dose ranges based on previous reports of differential effects between low- and high-dose (poly)phenols in vivo ${ }^{(35)}$. The most potent anti-inflammatory compounds from our experiments in Jurkat $\mathrm{CD}^{+}{ }^{+} \mathrm{T}$-lymphocytes were isorhamnetin (a flavonol that occurs as a glycoside in apples, onions and green tea), curcumin (from the Indian spice turmeric) and resveratrol (which is present in the skin of red, purple and black grapes and in especially high concentrations in Itadori tea).

The low molecular weight phenolic acids (Fig. 2) were generally less effective at reducing cytokine release than the unconjugated polyphenols, although some reductions in Il-2 release were seen with vanillic acid, tyrosol and 4-hydroxymandelic acid. Vanillic acid is a catabolite of multiple dietary polyphenols, including those found in wheat and blackcurrant juice ${ }^{(36)}$, and is also present at high concentrations in açai berries ${ }^{(37,38)}$. Vanillic acid significantly reduced IL-2 release from non-stimulated Jurkat $\mathrm{CD}^{+}{ }^{+}$-lymphocytes at $1 \mu \mathrm{mol} / \mathrm{l}$, which is within the range of concentrations previously reported in human plasma ${ }^{(36)}$, suggesting that vanillic acid may be a physiologically relevant antiinflammatory metabolite of dietary polyphenols.

Following ingestion of (poly)phenol-rich foodstuffs, a range of (poly)phenols, conjugates and catabolites are absorbed into the circulation. It is not understood whether the exposure of $\mathrm{CD}^{+}{ }^{+} \mathrm{T}$-lymphocytes to mixtures of these compounds may elicit different responses in comparison with the same compounds applied individually. To begin to address this issue, we treated 
(a)

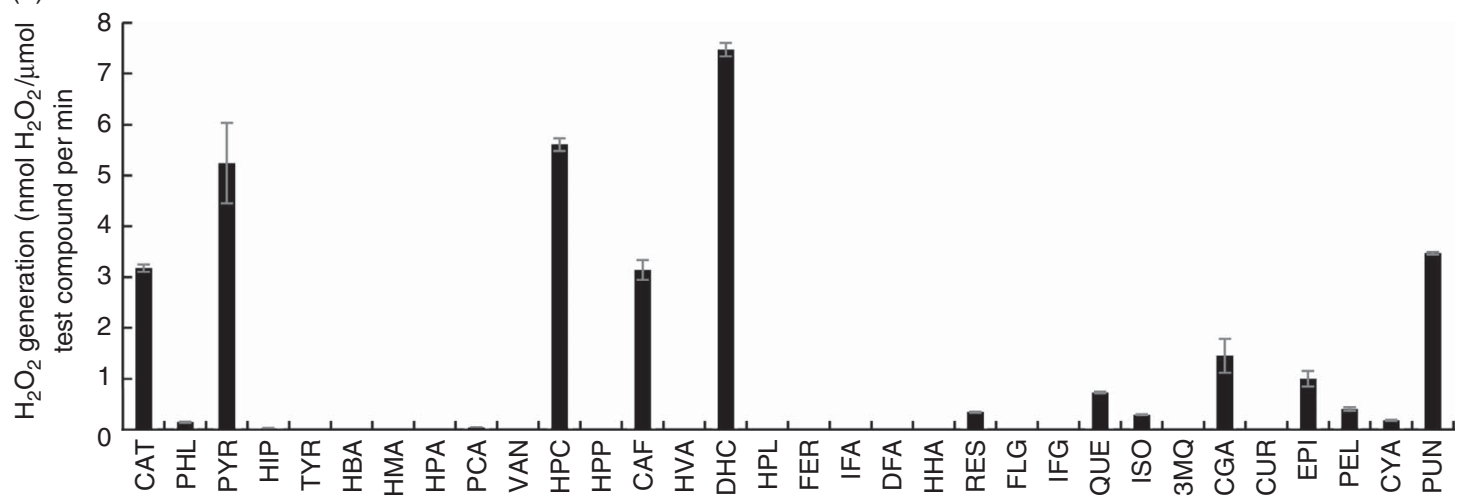

(b)

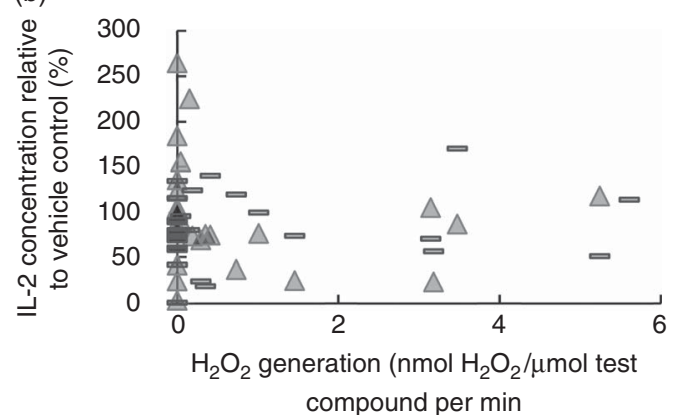

(d)

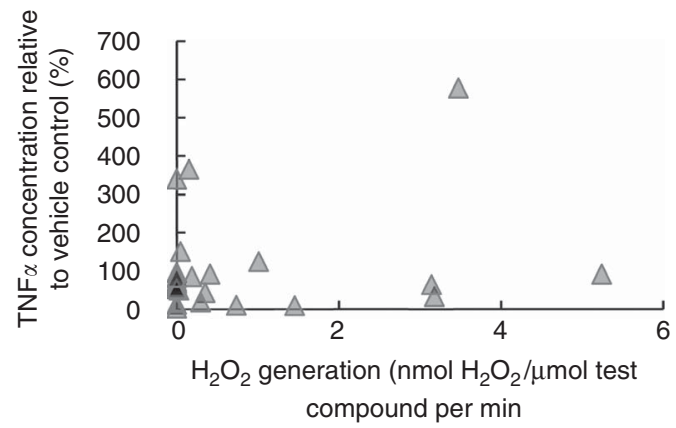

(c)

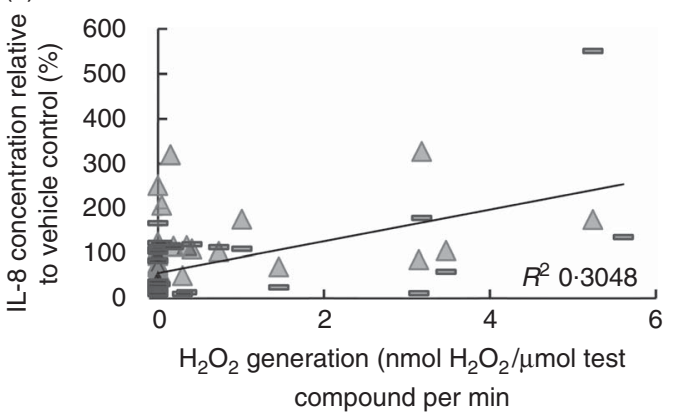

(e)

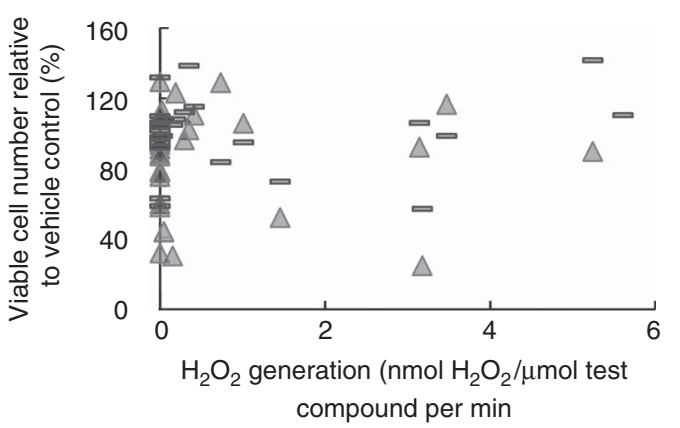

Fig. 6. Generation of $\mathrm{H}_{2} \mathrm{O}_{2}$ by (poly)phenols at $30 \mu \mathrm{mol} / \mathrm{l}$ concentration in Roswell Park Memorial Institute (RPMI)-1640 medium containing $10 \%$ fetal calf serum and phenol red (a), and relationships with Jurkat $\mathrm{CD}_{4}^{+}$T-lymphocyte pro-inflammatory cytokine release and cell growth (b-e). $\mathrm{H}_{2} \mathrm{O}_{2}$ production was measured by a kinetic reaction between each (poly)phenol incubated at $30 \mu \mathrm{mol} / \mathrm{l}$ with Amplex red reagent, which fluoresces following reaction with $\mathrm{H}_{2} \mathrm{O}_{2}$ (a). Scatter plots were constructed for $\mathrm{H}_{2} \mathrm{O}_{2}$ production against Jurkat CD4 ${ }^{+}$T-lymphocyte cytokine release ( $b=\mathrm{IL}-2, \mathrm{c}=\mathrm{IL}-8, \mathrm{~d}=\mathrm{TNF} a$ ) or cell number (e) after $48 \mathrm{~h}$ of treatment, with or without phorbol 12-myristate 13-acetate (PMA)/phytohaemagglutinin (PHA) stimulation to induce cytokine release at the $24 \mathrm{~h}$ time point. A linear correlation was identified between $\mathrm{H}_{2} \mathrm{O}_{2}$ production and IL-8 release in non-stimulated cells only $\left(R^{2} 0.3048\right.$; trend line shown in figure). 3MQ, 3-O-methylquercetin; CAF, caffeic acid; CAT, catechol; CGA, chlorogenic acid; CUR, curcumin; CYA, cyanidin-3-O-glucoside; DHC, dihydrocaffeic acid; DFA, dihydroferulic acid; EPI, (-)-epigallocatechin-3-O-gallate; FER, ferulic acid; FLG, feruloylglycine; HBA, 4-hydroxybenzoic acid; HHA, 4'-hydroxyhippuric acid; HIP, hippuric acid; HMA, 4'-hydroxymandelic acid; HPA, 4'-hydroxyphenylacetic acid; HPC, homoprotocatechuic acid; HPL, 3-(4'-hydroxyphenyl)lactic acid; HPP, 5-(3'-hydroxyphenyl)propionic acid; HVA, homovanillic acid; IFA, isoferulic acid; IFG, isoferuloylglycine; ISO, isorhamnetin; PCA, protocatechuic acid; PEL, pelargonidin-3-O-glucoside; PHL, phloroglucinol; PUN, punicalagin; PYR, pyrogallol; QUE, quercetin; RES, resveratrol; TYR, tyrosol; VAN, vanillic acid. Compounds are ordered from least (CAT) to highest (PUN) molecular weight along the $x$-axis. $\mathbf{\square}$, Data from non-stimulated cells; $\mathbf{\Lambda}$, data from PMA/PHA-stimulated cells.

Jurkat CD4 ${ }^{+}$T-lymphocytes with six different mixtures of (poly) phenols. The mixtures of compounds used were selected as representative of low molecular weight colonic metabolites (mix 1), mid-molecular weight colonic catabolites (mix 2), dietary polyphenols (mix 3), polyphenols and high-molecular weight hydroxycinnamate metabolites (mix 4), hydroxycinnamate derivatives derived from chlorogenic acid produced after the consumption of coffee (mix 5) and compounds potentially derived from apples (mix 6). Of these, five mixtures reduced cytokine release more than that predicted based on the cumulative effects of individual compounds. In contrast, mixture 1 was found to be relatively pro-inflammatory and comprised low molecular weight catabolites that individually induced pro-inflammatory cytokine release. Thus, we speculate that there may be synergistic anti-inflammatory effects of some polyphenols when they are present in foodstuffs, and 
the data obtained generally support this. Demonstration of true synergy between bioactive materials such as drugs requires a rigorous statistical approach ${ }^{(39)}$, which we do not have the dose-response data to undertake for the large group of polyphenols that were studied; however, the data presented appear to indicate the possibility of such synergistic effects and warrant further investigation.

It has been reported that (poly)phenols produce $\mathrm{H}_{2} \mathrm{O}_{2}$ in cell culture media due to autoxidation catalysed by transition metals such as $\mathrm{Fe}$ and $\mathrm{Cu}$ and that this may influence the responses of cells in culture ${ }^{(17,40)}$. We measured the production of $\mathrm{H}_{2} \mathrm{O}_{2}$ by different (poly)phenols and related compounds in RPMI-1640 medium containing 10\% FBS using the Amplex red-HRP technique. This assay is specific for $\mathrm{H}_{2} \mathrm{O}_{2}$ in simple solutions and has been widely used to examine $\mathrm{H}_{2} \mathrm{O}_{2}$ release by cells and sub-cellular fractions of cells ${ }^{(41)} \cdot \mathrm{H}_{2} \mathrm{O}_{2}$ generation was found to vary between compounds, with certain phenolic catabolites producing relatively large amounts of $\mathrm{H}_{2} \mathrm{O}_{2}$ and others producing no detectable $\mathrm{H}_{2} \mathrm{O}_{2}$. Analysis of $\mathrm{H}_{2} \mathrm{O}_{2}$ generation by (poly) phenols in comparison with their chemical structures indicated that, in accordance with theoretical predictions ${ }^{(40)}$, molecules with orthohydroxy groups on adjacent carbons of a benzene ring tended to generate $\mathrm{H}_{2} \mathrm{O}_{2}$. However, protocatechuic acid and 3-O-methylquercetin, which have benzene ring orthohydroxy groups, generated negligible $\mathrm{H}_{2} \mathrm{O}_{2}$, and moderate $\mathrm{H}_{2} \mathrm{O}_{2}$ generation was detected from isorhamnetin and resveratrol, which do not have orthohydroxy groups, implying that other aspects of the chemical structure also influence $\mathrm{H}_{2} \mathrm{O}_{2}$ generation. The average rate of $\mathrm{H}_{2} \mathrm{O}_{2}$ generation for these compounds in RPMI- 1640 medium was $5.6 \mathrm{nmol} \mathrm{H}_{2} \mathrm{O}_{2} / \mu \mathrm{mol}$ (poly)phenol per min. We investigated whether $\mathrm{H}_{2} \mathrm{O}_{2}$ generation by each test compound correlated with their effects on the release of IL-2, IL- 8 or TNF $\alpha$ by Jurkat $\mathrm{CD}^{+}$ T-lymphocytes or the growth of Jurkat cultures. A correlation was identified between $\mathrm{H}_{2} \mathrm{O}_{2}$ production and IL- 8 release by $\mathrm{CD}^{+}{ }^{+}$T-lymphocytes that were not stimulated with PMA/PHA. Interestingly, previous studies have indicated that IL-8 production is subject to modulation by redox signalling ${ }^{(34)}$ and can be induced by extracellular $\mathrm{H}_{2} \mathrm{O}_{2}$ in epithelial cell lines via activation of the redox-sensitive transcription factors activator protein-1 (AP-1) and NF- $\kappa \mathrm{B}^{(42,43)}$. Our results suggest that with PMA/PHA stimulation the influence of $\mathrm{H}_{2} \mathrm{O}_{2}$ on IL-8 release appears to become negligible. IL-2 and TNF $\alpha$ release and cell proliferation showed no clear relationship with $\mathrm{H}_{2} \mathrm{O}_{2}$ generation in non-stimulated or PMA/PHA-stimulated Jurkat $\mathrm{CD}^{+}$T-lymphocytes. Halliwell et al. ${ }^{(44,45)}$ have also pointed out that the absence of any detection of $\mathrm{H}_{2} \mathrm{O}_{2}$ generation by a compound in cell culture cannot be equated to the stability of the compound as some polyphenols were observed to rapidly degrade and autoxidise in the absence of detectable $\mathrm{H}_{2} \mathrm{O}_{2}$ generation. Thus, our studies indicate a lack of correlation between in vitro $\mathrm{H}_{2} \mathrm{O}_{2}$ generation and effects of the compound on cytokine release, but do not exclude other cell culture artifacts that may have influenced the data obtained.

We chose to undertake a limited proof-of-principle study to investigate the potential anti-inflammatory effects of isorhamnetin, curcumin and resveratrol in primary PBMC- derived human lymphocytes. Cells were pre-treated with 1 or $30 \mu \mathrm{M}$ polyphenol for $5 \mathrm{~h}$, followed by stimulation of cytokine release using LPS stimulation and incubation for a total period of $24 \mathrm{~h}$. We observed a significant reduction in IP-10 release following treatment with $1 \mu \mathrm{M}$ isorhamnetin, and there was also a trend $(P=0.07)$ towards reduced TNF $\alpha$ release with the same treatment. These studies were undertaken using lymphocytes from a single donor and clearly require confirmation using cells from a larger group of donors, but this approach was considered out with the remit of the present study due to the large variability in cytokine release from lymphocytes obtained from different donors.

In conclusion, we have shown that (poly)phenols modulate the release of cytokines by Jurkat $\mathrm{CD}^{+}$T-lymphocytes under resting and chemically activated conditions. Some compounds were found to have anti-inflammatory effects, while others were pro-inflammatory, and the effects varied with dose, suggesting that some dietary (poly)phenols may be beneficial for the prevention or management of chronic inflammatory conditions. We identified isorhamnetin, curcumin, resveratrol and vanillic acid as negative regulators of pro-inflammatory cytokine release in Jurkat $\mathrm{CD}^{+}$ T-lymphocytes, and also showed that $1 \mu \mathrm{M}$ isorhamnetin reduced pro-inflammatory cytokine release by primary human lymphocytes. Use of mixtures of (poly)phenols also suggested that they may act synergistically to modulate cytokine release by Jurkat $\mathrm{CD}^{+}$T-lymphocytes. Taking into account the main dietary sources of the compounds, our data support a potential anti-inflammatory role for (poly)phenols derived from red, purple and black grapes, turmeric, whole wheat, blackcurrants, apples and onions, and suggest that individuals at risk of chronic inflammation, such as older people, may benefit from supplementing their diets with isorhamnetin, resveratrol, curcumin and vanillic acid or with food sources that yield these bioactive molecules.

\section{Acknowledgements}

The authors are grateful to Melanie Sinclair for the excellent technical support.

This work was supported by a grant from the Biotechnology and Biological Sciences Research Council (BBSRC) through the BBSRC Diet Research Industry Club, grant no. BB/I005994/1. The funder had no role in the design, analysis or writing of this article.

M. J. J., A. M. A., A. C., S. B. L. and F. Mc. A. were involved in formulating the research question and designing the study; C. T. F. and S. R. carried out the study; C. T. F., S. R., M. J. J., A. M. A. and S. B. L. analysed the data; C. T. F. wrote the first draft of the manuscript; and all the authors edited the final version of the manuscript.

S. B. L. is employed by Unilever plc. None of the authors has any conflicts of interest to declare.

\section{Supplementary material}

For supplementary material/s referred to in this article, please visit http://dx.doi.org/10.1017/S0007114516000805 


\section{References}

1. Zhang X, Shu XO, Xiang YB, et al. (2011) Cruciferous vegetable consumption is associated with a reduced risk of total and cardiovascular disease mortality. Am J Clin Nutr $\mathbf{9 4}$, 240-246.

2. McCullough ML, Peterson JJ, Patel R, et al. (2012) Flavonoid intake and cardiovascular disease mortality in a prospective cohort of US adults. Am J Clin Nutr 95, 454-464.

3. Nagura J, Iso H, Watanabe Y, et al. (2009) Fruit, vegetable and bean intake and mortality from cardiovascular disease among Japanese men and women: the JACC Study. Br J Nutr 102, 285-292.

4. Jing Y, Han G, Hu Y, et al. (2009) Tea consumption and risk of type 2 diabetes: a meta-analysis of cohort studies. J Gen Intern Med 24, 557-62.

5. Carter P, Gray LJ, Troughton J, et al. (2010) Fruit and vegetable intake and incidence of type 2 diabetes mellitus: systematic review and meta-analysis. Biomed J 341, c4229.

6. Luo J, Gao YT, Chow WH, et al. (2010) Urinary polyphenols and breast cancer risk: results from the Shanghai Women's Health Study. Breast Cancer Res Treat 120, 693-702.

7. Rossi M, Lugo A, Lagiou P, et al. (2011) Proanthocyanidins and other flavonoids in relation to pancreatic cancer: a case-control study in Italy. Ann Oncol 23, 1488-1493.

8. Yang G, Zheng W, Xiang YB, et al. (2011) Green tea consumption and colorectal cancer risk: a report from the Shanghai Men's Health Study. Carcinogenesis 32, 1684-1688.

9. Zhong L, Goldberg MS, Gao YT, et al. (2001) A populationbased case-control study of lung cancer and green tea consumption among women living in Shanghai, China. Epidemiology 12, 695-700.

10. Dai Q, Borenstein AR, Wu Y, et al. (2006) Fruit and vegetable juices and Alzheimer's disease: the Kame Project. Am J Med 119, 751-759.

11. Tan LC, Koh WP, Yuan JM, et al. (2008) Differential effects of black versus green tea on risk of Parkinson's disease in the Singapore Chinese Health Study. Am J Epidemiol 167, $553-560$

12. Chiva-Blanch G, Urpi-Sarda M, Llorach R, et al. (2012) Differential effects of polyphenols and alcohol of red wine on the expression of adhesion molecules and inflammatory cytokines related to atherosclerosis: a randomized clinical trial. Am J Clin Nutr 95, 326-334.

13. Tomé-Carneiro J, Gonzálvez M, Larrosa M, et al. (2012) One-year consumption of a grape nutraceutical containing resveratrol improves the inflammatory and fibrinolytic status of patients in primary prevention of cardiovascular disease. Am J Cardiol 110, 356-363.

14. Karlsen A, Paur I, Bøhn SK, et al. (2010) Bilberry juice modulates plasma concentration of NF- $\mathrm{KB}$ related inflammatory markers in subjects at increased risk of CVD. Eur J Nutr $\mathbf{4 9}$ 345-355.

15. Park S-J, Ahmad F, Philp A, et al. (2012) Resveratrol ameliorates aging-related metabolic phenotypes by inhibiting cAMP phosphodiesterases. Cell 148, 421-433.

16. Akagawa M, Shigemitsu T \& Suyama K (2003) Production of hydrogen peroxide by polyphenols and polyphenol-rich beverages under quasi-physiological conditions. Biosci Biotechnol Biochem 67, 2632-2640.

17. Long LH, Hoi A \& Halliwell B (2010) Instability of, and generation of hydrogen peroxide by, phenolic compounds in cell culture media. Arch Biochem Biophys 501, 162-169.

18. Scalbert A \& Williamson G (2000) Dietary intake and bioavailability of polyphenols. J Nutr 130, 2073S-2085S.
19. Vitaglione P, Barone Lumaga R, Ferracane R, et al. (2013) Human bioavailability of flavanols and phenolic acids from cocoa-nut creams enriched with free or microencapsulated cocoa polyphenols. Br J Nutr 109, 1832-1843.

20. Del Rio D, Rodriguez-Mateos A, Spencer J, et al. (2013) Dietary (poly)phenolics in human health: structures, bioavailability, and evidence of protective effects against chronic diseases. Antioxid Redox Signal 18, 1818-1892.

21. Rechner AR, Smith MA, Kuhnle G, et al. (2004) Colonic metabolism of dietary polyphenols: influence of structure on microbial fermentation products. Free Radic Biol Med 36, 212-225.

22. Rodriguez-Mateos A, Vauzour D, Krueger CG, et al. (2014) Bioavailability, bioactivity and impact on health of dietary flavonoinds and related compounds: an update. Arch Toxicol 88, 1803-1853.

23. Bao B, Prasad AS, Beck FW, et al. (2003) Zinc modulates mRNA levels of cytokines. Am J Physiol Endocrinol Metab 285, E1095-E1102.

24. Ader P, Wessmann A \& Wolffram S (2000) Bioavailability and metabolism of the flavonol quercetin in the pig. Free Radic Biol Med 28, 1056-1067.

25. Dong Y, Guha S, Sun X, et al. (2012) Nutraceutical interventions for promoting healthy aging in invertebrate models. Oxid Med Cell Longev 2012, e718491.

26. Loke WM, Jenner AM, Proudfoot JM, et al. (2009) A metabolite profiling approach to identify biomarkers of flavonoid intake in humans. J Nutr 139, 2309-2314.

27. Woodward GM, Needs PW \& Kay CD (2011) Anthocyaninderived phenolic acids form glucuronides following simulated gastrointestinal digestion and microsomal glucuronidation. Mol Nutr Food Res 55, 378-386.

28. Sanbongi C, Suzuki N \& Sakane T (1997) Polyphenols in chocolate, which have antioxidant activity, modulate immune functions in humans in vitro. Cell Immunol 177, 129-136.

29. Mao TK, Powell J, Van de Water J, et al. (2000) The effect of cocoa procyanidins on the transcription and secretion of interleukin 1 beta in peripheral blood mononuclear cells. Life Sci 66, 1377-1386.

30. Gupta SC, Tyagi AK, Deshmukh-Taskar P, et al. (2014) Downregulation of tumor necrosis factor and other proinflammatory biomarkers by polyphenols. Arch Biochem Biophys 559, 91-99.

31. Siriwardhana N, Kalupahana NS, Cekanova M, et al. (2013) Modulation of adipose tissue inflammation by bioactive food compounds. J Nutr Biochem 24, 613-623.

32. Crozier A, Del Rio D \& Clifford MN (2010) Bioavailability of dietary flavonoids and phenolic compounds. Mol Aspects Med 31, 446-467.

33. Roowi S, Stalmach A, Mullen W, et al. (2010) Green tea flavan3-ols: colonic degradation and urinary excretion of catabolites by humans. J Agric Food Chem 58, 1296-1304.

34. Yamaguchi T, Hiromasa K, Kabashima-Kubo R, et al. (2013) Nakamura $M$. galectin-7, induced by cis-urocanic acid and ultraviolet B irradiation, down-modulates cytokine production by T-lymphocytes. Exp Dermatol 22, 840-842.

35. Baron-Menguy C, Bocquet A, Guihot AL, et al. (2007) Effects of red wine polyphenols on postischemic neovascularization model in rats: low doses are proangiogenic, high doses anti-angiogenic. FASEB J 21, 3511-3521.

36. Mateo Anson N, Aura AM, Selinheimo E, et al. (2011) Bioprocessing of wheat bran in whole wheat bread increases the bioavailability of phenolic acids in men and exerts antiinflammatory effects ex vivo. J Nutr 141, $137-143$. 
37. Rechner AR, Kuhnle G, Hu H, et al. (2002) The metabolism of dietary polyphenols and the relevance to circulating levels of conjugated metabolites. Free Radic Res 36, 1229-1241.

38. Pacheco-Palencia LA, Mertens-Talcott S \& Talcott ST (2008) Chemical composition, antioxidant properties, and thermal stability of a phytochemical enriched oil from açai (Euterpe oleracea Mart.). J Agric Food Chem 56, 4631-4636.

39. Tallarida RJ (2011) Quantitative methods for assessing drug synergism. Genes Cancer 2, 1003-1008.

40. Perron N \& Brumaghim J (2009) A review of the antioxidant mechanisms of polyphenol compounds related to iron binding. Cell Biochem Biophys 53, 75-100.

41. Vasilaki A, Mansouri A, Van Remmen H, et al. (2006) Free radical generation by skeletal muscle of adult and old mice: effect of contractile activity. Aging Cell 5, 109-117.
42. Simeonova PP, Leonard S, Flood L, et al. (1999) Redoxdependent regulation of interleukin- 8 by tumor necrosis factor-alpha in lung epithelial cells. Lab Invest 79, 1027-1037.

43. Lakshminarayanan V, Drab-Weiss EA \& Roebuck KA (1998) $\mathrm{H}_{2} \mathrm{O}_{2}$ and tumor necrosis factor- $\alpha$ induce differential binding of the redox-responsive transcription factors AP-1 and NF- $\kappa \mathrm{B}$ to the interleukin-8 promoter in endothelial and epithelial cells. J Biol Chem 273, 32670-32678.

44. Long LH, Hoi A \& Halliwell B (2010) Instability of, and generation of hydrogen peroxide by, phenolic compounds in cell culture media. Arch Biochem Biophys 501, 162-169.

45. Long LH, Clement MV \& Halliwell B (2000) Artifacts in cell culture: rapid generation of hydrogen peroxide on addition of (-)-epigallocatechin, (-)-epigallocatechin gallate, (+)-catechin, and quercetin to commonly used cell culture media. Biochem Biophys Res Commun 273, 50-53. 